\title{
REMARQUES SUR LE CYCLE ÉVOLUTIF DES HÉMIURIDES
}

\author{
Par Robert-Ph. DOLLFUS
}

\section{Note préliminaire}

On sait depuis quelques années que, parmi les Hémiurides, il y a, au point de vue du cycle évolutif, d'une part les espèces qui sont enkystées au stade metacercaria, d'autre part les espèces ne sécrétant jamais de kyste : leur métacercaire se trouve libre dans leur hôte ou dans le plancton.

Comme exemples de larves s'enkystant, on peut citer celles de : Lecithochirium gravidum Looss (la métacercaire est progénétique), Lecithochirium rufoviride Rudolphi (la métacercaire est progénétique) et Synaptobothrium caudiporum Rudolphi.

Ces trois espèces appartiennent à la sous-famille des Sterrhurinæ Looss, leurs métacercaires sont enkystées dans divers téléostéens.

Les observations de larves d'Hémiurides non enkystées au stade metacercaria et trouvées libres soit dans le plancton, soit chez des animaux pélagiques sont extrêmement nombreuses.

Pour un certain nombre, les descriptions ne sont pas suffisamment précises pour qu'il soit actuellement possible de reconnaître quel est l'adulte correspondant. Cela tient en partie à ce que beaucoup d'observateurs, n'étant pas spécialistes, ne pouvaient avoir sur la morphologie de ces parasites les connaissances suffisantes; en partie aussi à ce qu'ils ont étudié des matériaux mal conservés; cela tient encore à ce que la systématique des Hémiurides est difficile et d'une acquisition relativement très récente, postérieure à beaucoup des observations.

Quoi qu'il en soit, celles des métacercaires du plancton et des animaux planctoniques qui ont pu être identifiées se rapportent, généralement, à la sous-famille des Hemiurinæ (Looss sensu, 1907) (1).

Je rappellerai très sommairement quelques observations de métacercaires non enkystées d'Hémiurides.

a. - F. Will (1844), à Trieste, dans les canaux aquifères de Beroe rufescens Forskål (Cténophore Nudicténide): Distoma beroes F. Will.

(1) Des métacercaires de Derogenina se trouvent semblablement chez les animaux pélagiques : on a identifié la métacercaire de Derogenes varicus $\mathrm{O}$. F. M. chez les Copépodes des côtes atlantiques du Canada (J. Stafford, 1905) et chez des Sagitta des côtes de Grande-Bretagne (M. V. Lebour, 1917).

Les Derogenina forment une sous-famille dans la famille des Syncœliida, celle-ci faisant partie de la superfamille des Hemiurida.

Annales de Parasitologie, T. I, No 4 . - Octobre 1923. 
b. - J. Müller (1850) aurait retrouvé D. beroes $\mathrm{F}$. W. dans le plancton, libre.

c. - Busch (1851), à Trieste, chez les Sagitta (Chætognathes), observa plusieurs espèces de métacercaires dont une d'Hémiuride $(D$. beroes F. W.) et une d'Accacœlida (Distoma fimbriatum Busch) (1).

d. - G. Wagener (1860) a signalé de petites formes immatures à postabdomen rétracté dans des céphalopodes et autres animaux marins Ínférieurs de la Méditerranée ; il crut qu'il s'agissait de Distoma rufoviride Rud.

e. - Claus (1863) a étudié une forme larvaire libre dans la cavité générale de Paracalanus parvus Claus, il est probable qu'il s'agissait d'une larve d'Hémiuride.

f. - Willemoes-Suhm (1871), dans la mer Baltique, larves libres dans le plancton et fixées par leur acetabulum sur des copépodes et larves d'Annélides; larve à moitié engagée dans le corps d'un copépode.

g. - Uljanine (1871), dans la Mer Noire, larves libres dans le plancton et fixées sur des copépodes.

h. - Grebnitzky (1873), Distoma ichthyophorba Grebn., libre dans Ichthyophorba angustata Claus (= Centropages hamatus Lillj.) de la mer Noire.

i. - Giesbrecht (1882), à Kiel, larves libres dans le plancton, complètement enfermées ou à moitié engagées dans Lucullus acuspes Giesb. (=Pseudocalanus elongatus Bøeck) et Centropages hamatus Lillj.

j. - Pouchet (1883), à Concarneau, dans Noctiluca.

k. - Pouchet et de Guerne (1887), à Concarneau dans le plancton et Noctiluca.

l. - Monticelli (1891), jeune «Apoblema » fixé sur la peau d'un pleuronecte.

m. - E. Canu (1892), côtes du Boulonnais, dans le plancton et dans Paracalanus parvus Claus et Acartia Clausi Giesbrecht.

n. - H.-S. Pratt (1898), larves libres dans le plancton et engagées dans des copépodes (Acartia?) du Long Island Sound.

o. - J. Stafford (1900), Distoma pelagicum Stafford, libres dans le plancton du Nouveau-Brunswick.

p. - E. Chatton (1908), à Banyuls-sur-Mer, dans les Acartia.

q. - Monticelli (1908), larves d'Aphanurus chez les Sagitta, à Naples.

$r$. - Steuer (1908 [in Monticelli] et 1910), larves d'Aphanurus chez les Sagitta du golfe de Trieste, Rovigno.

s. - D.-Th. Sinitzine (1911), Adolescaria appendiculata Sin. dans des Sagitta et copépodes de la mer Noire.

t. - Apstein (1911), mer du Nord et mer Baltique, dans Calanus fin. marchicus Gunn., Pseudocalanus elongatus Bæek, Paracalanus parvus Claus, Temora longicornis O. F. M., Centropages hamatus Lillj., Acartia sp.

(1) Nous ne traiterons pas ici la question des métacercaires d'Accacoelidæ trouvées libres de tout kyste chez des animaux planctoniques, rappelons toutefois que la famille des Accacoelide fait partie de la superfamille des Hemiurida. 
u. - Cooper (1915), dans divers copépodes, dont Acartia Clausi Giesb., de la còte atlantique du Canada.

v. - Maurice Rose (observations inédites, 1921 et 1922), à Roscoff, chez Acartia Clausi Giesb., Centropages hamatus Lillj., Temora longicornis O. F. M., Centropages typicus Kröyer (voir les figures 1 et 2).

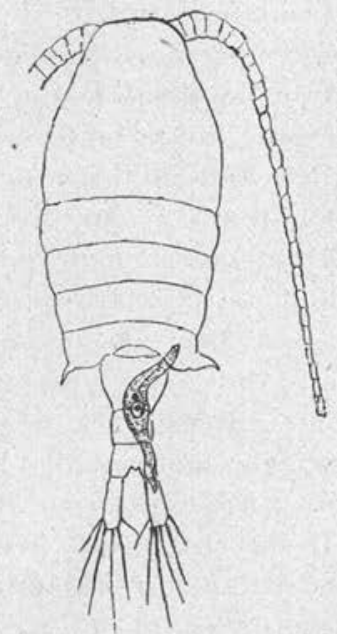

Fig. 1. - Centropages hamatus Lillj. contenant une métacercaire libre dans l'abdomen. (Maurice Rose inven. et delin., Roscoff, 1921).

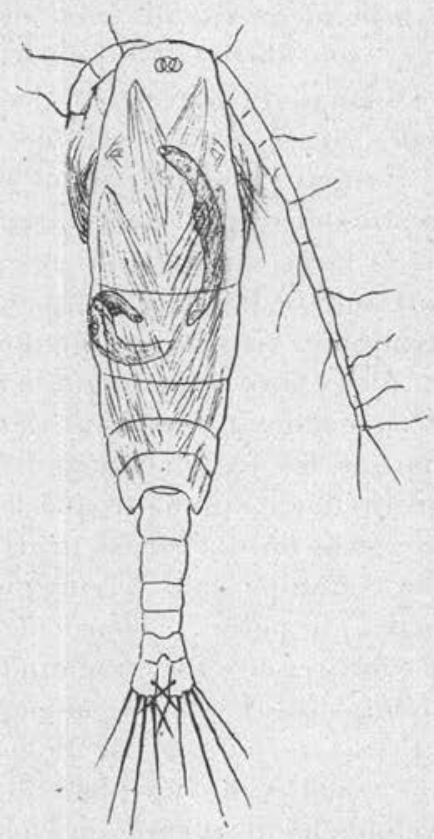

Fig. 2. - Acartia Clausi Giesbrecht, mâle contenant deux métacercaires libres dans la cavité générale sous la musculature. (Maurice Rose inven. et delin., Roscoff, 1921).

w. - Th. Monod (observation inédite), libre dans le plancton de la région de Concarneau (R.-Ph. Dollfus et Th. Monod leg, 1921).

$x$. - M. V. Lebour (1923), larve d'Hemiurus communis Odhner, deux fois chez Acartia Clausi Giesb. (une fois chez un mâle, une fois chez une femelle), dans la région de Plymouth.

L’identification spécifique de la larve étudiée par M. V. Lebour ne paraît pas pouvoir être mise en doute et nous nous accordons avec Odhner (1905) et M. V. Lebour (1923) pour considérer la larve étudiée par Pratt (1908) comme devant être rapportée à Hemiurus communis Odhner ou tout au moins à une espèce extrêmement voisine, bien que Pratt n'ait pas décrit la vésicule séminale comme formée de deux lobes. 
Nous nous abstenons à dessein de rappeler ici (1) et de discuter beaucoup de déterminations douteuses proposées par les observateurs de larves d'Hémiurides, disons seulement que, dans un grand nombre de cas, l'adulte correspondant a été supposé être Hemiurus appendiculatus Rud.

Au point de vue du cycle évolutif, Pratt a supposé que le miracidium, issu de l'œuf rejeté par le poisson, hôte définitif, devenait directement la larve que l'on trouve dans les copépodes, Sagitta, Beroe, etc..., ce miracidium rechercherait un copépode ou serait passivement absorbé par lui par voie digestive, il passerait du tube digestif dans la cavité générale, où il se transformerait, grandirait, puis la larve sortirait du copépode, devenant libre dans le plancton. Pratt rejette l'hypothèse, proposée par Willemoes-Suhm, de la pénétration, à travers les téguments du copépode, de la larve rencontrée libre dans le plancton, s'appuyant sur le fait qu'il a rencontré des copépodes renfermant des larves tout entières à leur intérieur, sans que les téguments monśrent trace de blessure ou de passage pouvant faire supposer que le parasite ait pénétré en traversant la paroi externe du corps. Pratt a, tout au contraire, observé que la larve contenue dans le copépode était susceptible, après des efforts répétés, de percer la paroi de son hôte par l'intérieur et de sortir. On capture, en effet, quelquefois, des copépodes portant des larves d'Hémiurides en partie engagées dans leur corps, en partie dépassant à l'extérieur (2) (Willemoes-Suhm, Giesbrecht, Pratt, Lebour). Dans le corps du copépode, la métacercaire atteint un maximum de développement qu'elle ne dépassera qu'en devenant adulte dans l'hôte définitif et l'on comprend que si le copépode est mourant ou ne peut plus lui fournir la nourriture dont elle a besoin, la métacercaire l'abandonne ; il peut cependant arriver que la métacercaire, ayant dévoré les parties molles de son hôte, y reste enfermée comme dans une boîte, ainsi que l'a observé Giesbrecht (1882).

Que le poisson, hôte définitif, s'infeste en absorbant soit directement la métacercaire mûre libérée dans le plancton, soit la métacercaire enclose dans le copépode ou dans un autre animal pélagique, cela ne semble pas douteux, mais il reste à connaitre la première partie du cycle évolutif, entre le miracidium et le stade metacercaria.

M. V. Lebour (comme Pratt) estime probable que le miracidium

(1) Nous reviendrons sur ce sujet dans une publication ultérieure.

(2) On remarquera, sur les figures données par Pratt et Lebour, par exemple, que c'est l'extrémitế antérieure de la larve d'Hemiurus qui est engagée dans le copépode ; l'extrémité postérieure sortirait la première. 
pénètre directement dans le copépode et s'y transforme, mais ajoute qu'il est aussi concevable qu'il existe un hôte antérieur dans lequel le miracidium devient sporocyste et il pourrait $\mathrm{y}$ avoir un stade rédie; le copépode dans ce cas serait seulement le deuxième hôte intermédiaire.

Nous estimons que la première hypothèse est inacceptable et qu'il n'y a pas de raison pour supposer les stades sporocyste, rédie, cercaire supprimés chez des Hémiurides.

Faisons tout d'abord remarquer que si, jusqu'à aujourd'hui, aucune des cercaires connues dans la faune marine n'a été considérée comme correspondant à un Hémiuride, on connait depuis longtemps dans la faune d'eau douce Cercaria cystophora Wagener (1866), qui est la larve d'Halipegus ovocaudatus Vulpian (1858) et le genre Halipegus appartient incontestablement à la superfamille des Hemiurida (1).

Le miracidium de l'œuf d'Halipegus pénètre chez divers Planorbis, y donne un sporocyste primitif produisant des rédies où naissent les cercaires. A leur sortie du Planorbe, les cercaires mùres comportent une queue à portion antérieure dilatée en forme de poche où peut se rétracter le corps proprement dit de la cercaire, cette poche contient aussi un long filament protractile. La cercaire, libérée dans le plancton d'eau douce, pénètre chez Calopteryx virgo L., vraisemblablement comme proie, elle y devient métacercaire dans la ca-ité générale, sans s'enkyster, ainsi que l'a observé Sinitzine (1905). C'est dans cet état qu'elle arrive dans l'hôte définitif mangeur de Calopteryx (Rana esculenta L., Rana temporaria L.) où elle devient adulte.

On connaît plus d'une douzaine d'espèces de cercaires du groupe de C. cystophora Wag., tant d'eau douce (chez des Pulmonés et des Pectinibranches) que marines (chez des Tectibranches, Ptéropodes et Pectinibranches) ; certaines d'entre elles sont capables de sécréter un kyste, d'autres n'en sécrètent pas.

Chez les Calliostoma conuloïdes Lamarck, de Roscoff (Finistère), nous a vons fréquemment trouré (1913), dans des rédies, une cercaire cystophore (Cercaria callioslomre nobis), asscz voisine de C. cystophora Wag., qui nous paraît être aussi une larve d'Hémiuride. Son étude histologique nous a montré qu'elle ne possédait pas de glandes kystogènes.

(1) Il doit être compris dans la famille des Syncoliida, étant étroitement apparenté au genre Syncoelium (il y a un canal de Laurer et pas de receptaculum seminis) et aux genres Derogenoỉdes Nicoll (même emplacement des glandes génitales) et Hemipera Nicoll (œufs pourvus d'un filament polaire). 


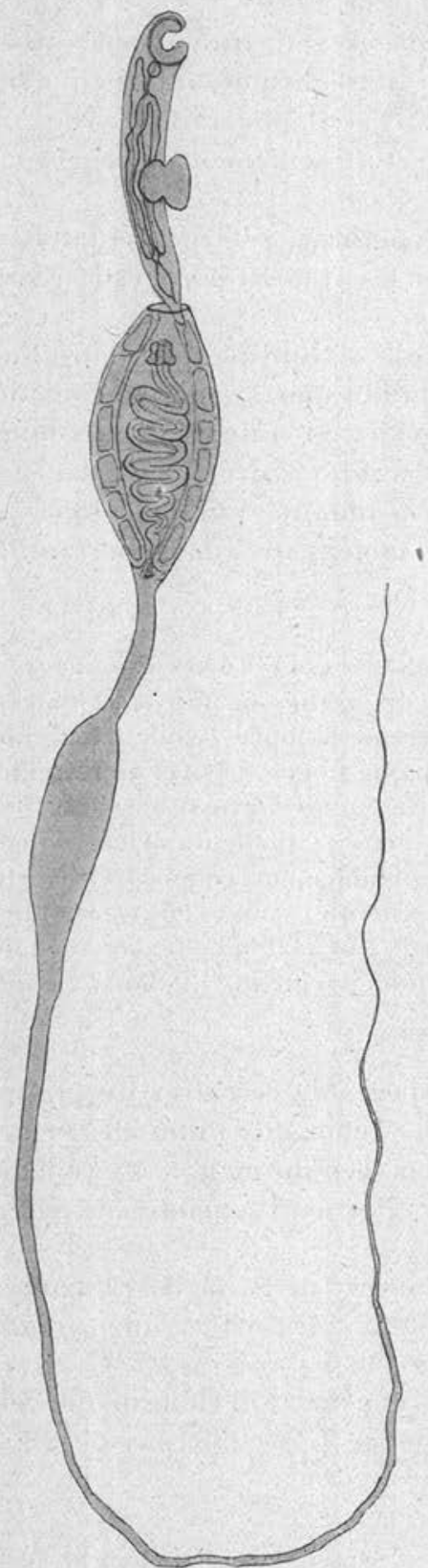

Fig. 3. - Cercaria calliostoma nob. (R.-Ph. Dollfus inven. et delin., Roscoff, 1913). Gr. env, 135.
Comme sa cuticule est striéc transversalement et que les branches latérales de la vessie s'anastomosent dorsalement au niveau de la ventouse orale, il s'agit vraisemblablement d'une espèce de la sousfamille des Hemiurinæ Looss 1907, qui comprend seulement les gen res Hemiurus et Aphanurus. Nou; ne pouvons préciser l'espèce adulte correspondante car notre cercaire n'avait pas encore d'ébauches bien délimitées de glandes génitales. mais nous pensons que cette cercaire, libérée dans le plancton, poursuit un cycle évolutif paral. lèle à celui de $C$. cystophora Wag. (1). Happée comme une proie par un copépode, elle y devient vraisemblablement, et sans enkystement, une des formes de métacercaires d'Hémiurides déjà connues chez les copépodes.

Diagnose de Cercaria calliostomæ $\mathbf{n}$. sp. - Corps allongé, arqué, un peu aplati dorso-ventralement, rétréci postérieurement, pourvu d'une ventouse orale et d'un acetabulum. Ventouse orale globuleuse, s'ouvrant un peu ventralement; acetabulum globuleux, profond, éversible, situé en arrière du milieu du corps, ayant un diamètre un

(1) Nous ne croyons pas que toutes les espéces de cercaires cystophores soient des cercaires d'Hémiurides, ni que chaque espèce d'Hémiuride corresponde à une cercaire cystophore, car des adultes très voisins peuvent avoir des cercaires à morphologie larvaire três différente et des cercaires à morphologie larvaire voisine, au moins en apparence, peuvent correspondre à des adultes de groupes très éloignés. Il est toujours utile cependant de rechercher les comparaisons morphologiques, quitte à les abandonner lorsqu'on s'apercoit qu'elles ne reposent que sur des apparences superficielles, c'est-à-dire seulement sur des caractères purement larvaires. 
peu plus grand que la ventouse antérieure. Cuticule striée transversalement. Prépharynx nul, pharynx sphérique, petit, œsophage aussi long que le pharynx, cœca intestinaux larges, s'étendant environ jusqu'à la limite entre les deux derniers septièmes de la longueur du corps. Vessie s'ouvrant à l'extrémité postérieure du corps, formée d'un tronc médian, impair, occupant environ le dernier septième de la longueur du corps, se bifurquant en deux branches latérales grêles qui se réunissent transversalement et dorsalement au voisinage de la ventouse orale, vers la fin du premier septième de la longueur du corps.

Queue environ sept fois plus longue que le corps, comprenant plusieurs parties. La partie antérieure, appelée poche intermédiaire, est ellipsoïdale, à grand axe ayaní à peu près la mème longueur que le corps; elle est creuse avec une double paroi et ouverte antérieurement; dans le bord ventral de l'ouverture s'insère l'extrémité postérieure du corps de la cercaire. Au fond de la poche s'insère un long filament protractile appelé flèche, mesurant environ 5 fois la longueur du corps. L'extrémité distale du filament est munie d'une sorte de manchon plus large que le reste du filament. Le corps de la cercaire et la flèche peuvent se rétracter entièrement dans la poche intermédiaire. A cette poche fait suite une région rétrécie, pleine, moins longue qu'elle, qui se continue par un renflement allongé, s'atténuant graduellement, la queue devenant filiforme jusqu'à son extrémité postérieure.

Rédıe longue, cylindriforme, munie antérieurement d'une fossette où s'ouvre le pharynx, régulièrement ellipsoïdal, auquel fait suite un court œsophage donnant dans la poche gastrique. Cavité générale vaste, où se développent les embryons de cercaires. Canal d'accouchement cilié, s'ouvrant antèrieurement à côté de la bouche.

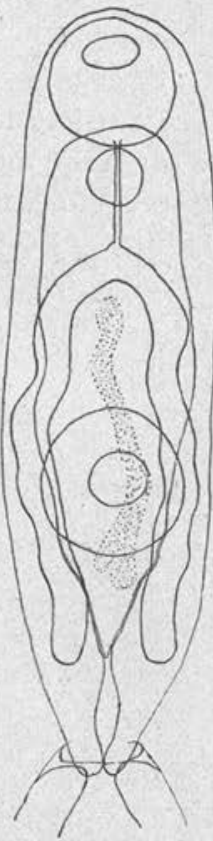

Fig. 4. - Corps proprement dit de la même cercaire; la striation transversale n'est pas représentée. Gr. env.375.

Dimensions approximatives d'une cercaire (vivante). Longuetr toiale : $2 \mathrm{~mm}$.; longueur du corps : $250 \mu$; largeur : $65 \mu$; épaisseur : $45 \mu$; longucur de la poche intermédiaire : $250 \mu$; largeur : $95 \mu$; longueur du reste de la queue : $1 \mathrm{~mm}$. 5 ; ventouse antérieure, diam. : $40 \mu$; acetabulum, diam. : $45 \mu$. Rédie (après fixation). Longueur : $4 \mathrm{~mm}$.; largeur moyenne : $300 \mu$; longueur du pharynx : $150 \mu$; diamètre : $70 \mu$; longueur de la poche gastrique : $800 \mu$.

Museum national d'histoire naturelle. (Laboratoire de M. le professeur A. Gruvel.) 